\title{
Improvement in Quality of Life after Myomectomy
}

Ehab Helmy Abdel Malek Fahmy ${ }^{1}$, Constance Liew Sat Lin², Alvin Oliver Payus²*, Rhanye Mac Guad³, May Zaw Soe ${ }^{1}$, Satishratnam Nagaratnam ${ }^{4}$, Symeon Mandrinos ${ }^{5}$, Chin Yeung Sing ${ }^{6}$, ABM Tofazzal Hossain ${ }^{7}$

${ }^{1}$ Department of Reproductive Health, Faculty of Medicine and Health Sciences, Universiti Malaysia Sabah, Kota Kinabalu, Sabah, Malaysia

2 Department of Medical-Based Discipline, Faculty of Medicine and Health Sciences, Universiti Malaysia Sabah,

Kota Kinabalu, Sabah, Malaysia

${ }^{3}$ Department of Biomedical Science and Therapeutics, Faculty of Medicine and Health Sciences, Universiti Malaysia Sabah, Kota Kinabalu, Sabah, Malaysia

${ }^{4}$ Labuan Hospital, Obstetrics and Gynaecology Department, Labuan, Malaysia

${ }^{5}$ Swinburne University of Technology Sarawak Campus, Kuching, Sarawak, Malaysia

${ }^{6}$ Sabah Women and Child Hospital, Gyn. Oncology Department, Kota Kinabalu, Sabah, Malaysia

${ }^{7}$ Department of Surgery, Faculty of Medicine and Health Sciences, Universiti Malaysia Sabah, Kota Kinabalu, Sabah, Malaysia

*Corresponding author's email: dralvinpayus@ums.edu.my

Received: 21 March 2019

Accepted: 25 October 2019

\section{Keywords:}

heavy menstrual bleeding, blood transfusion, dysmenorrhea, quality of life, myomectomy

\section{ABSTRACT}

Dysmenorrhea is one of the leading causes of pelvic pain and menstrual disorder among women during childbearing age. The burden of dysmenorrhea is greater than any other gynaecological complaint. Some women have severe dysmenorrhea which renders them incapacitated for days each menstrual cycle requiring absence from study or duty, frequently requiring pain killer, restriction of daily performance, poor sleep, negative moods such as anxiety and depression. A 31-year-old female presented with severe dysmenorrhea and heavy menstrual bleeding (HMB) as a cause of multiple uterine fibroids, underwent surgeries to remove 100 fibroids from her uterus which has improved her quality of life, eliminating her dysmenorrhea and menstrual abnormalities.

\section{INTRODUCTION}

The prevalence of dysmenorrhea is 45 to $93 \%$ of women during reproductive age. The primary dysmenorrhea is common in young women and remains a good prognosis even though it is associated with low quality of life. The secondary forms of dysmenorrhea are associated with endometriosis and adenomyosis ${ }^{1}$. Recent research suggests that prostaglandin F2a (PGF2a), a potent myometrial stimulant and vasoconstrictor in the secretory endometrium is the main pathogenesis of primary dysmenorrhea. The increase in prostaglandins in the endometrium after the fall in progesterone in the late luteal 
phase results in increased myometrial tone and excessive uterine contraction ${ }^{2}$.

\section{CASE PRESENTATION}

This 31-year-old lady visited a fertility clinic in 2009 due to subfertility for six years during her first and second marriage. Her menarche was at the age of 13 years and had regular menstrual cycles until the age of 20 years. The patient had a myomectomy surgery done in 2007 during which 20 fibroids have been removed. Infertility workout was done and her hysterosalpingogram showed partial bicornuate uterus with no endometrial split as reported in 2010. During her clinic followup in February 2011, it was noted that the patient had recurrent multiple uterine fibroids. Hysteroscopy was done in March 2011 and it was noted that she had multiple submucosal fibroids at the fundus and posterior wall. The patient was referred to a tertiary centre for transcervical resection of myoma (TCRM) in July 2011. At the tertiary centre, the patient was opted for conservative treatment after counselling with the doctor at the fertility clinic.

In March 2012, this patient presented with heavy menstrual bleeding for 3 days with symptom and signs of anaemia, her haemoglobin $(\mathrm{Hb})$ level was $5.11 \mathrm{~g} / \mathrm{dL}$. Upon admission to a specialist hospital, 4 pints of pack cells (PC) were transfused over 24 hours and her haemoglobin level increased to 11.5 $\mathrm{g} / \mathrm{dL}$ upon discharge. In June 2012, the patient presented with an episode of heavy menstrual bleed, with 10 - 15 pads fully soaked per day for 3 consecutive days. On admission, her haemoglobin level was $6.79 \mathrm{~g} / \mathrm{dL}$ and she was transfused 2 pints pack cells and her $\mathrm{Hb}$ improved to $9.11 \mathrm{~g} / \mathrm{dL}$.

Ultrasound scanning was done on the same day which showed multiple uterine fibroids measuring $3-6 \mathrm{~cm}$ (Figure 1).

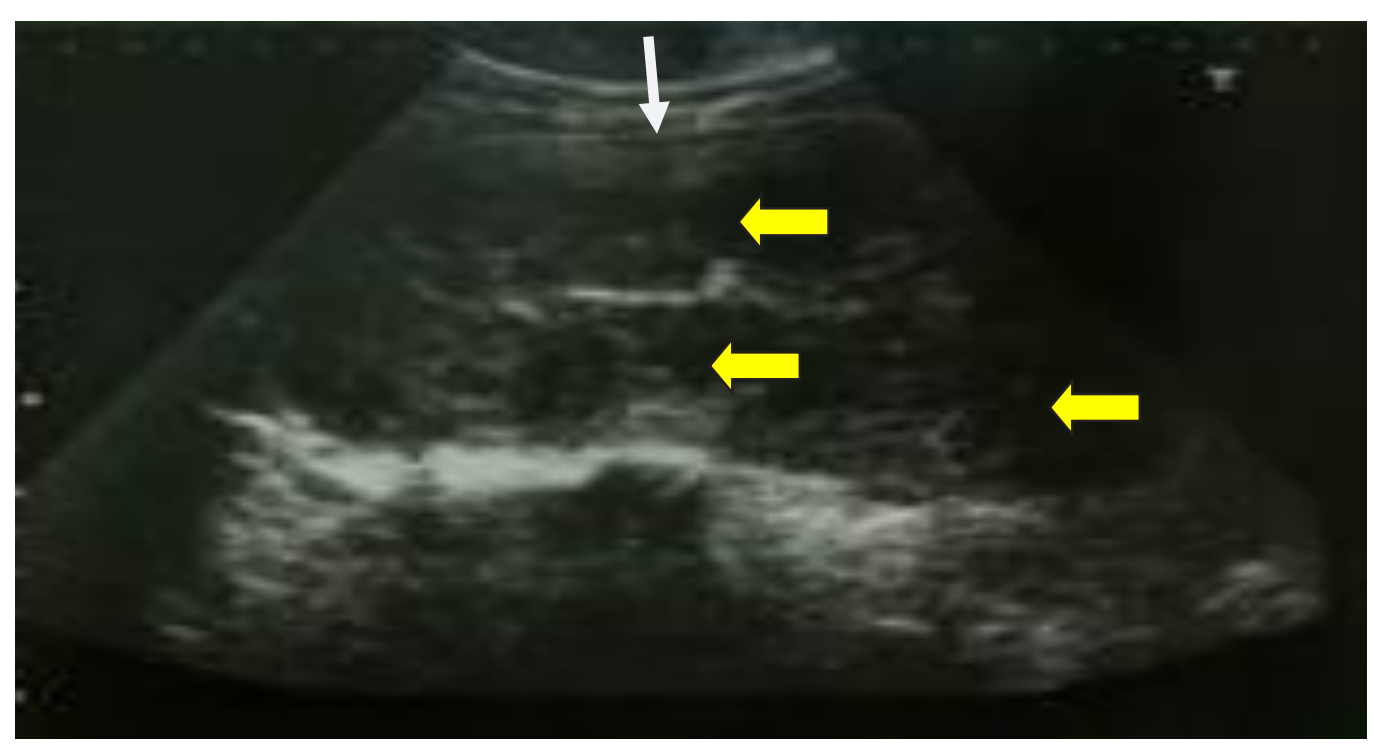

Figure 1 Pre-operative scan. Yellow arrows indicate multiple uterine fibroids measuring $3-6 \mathrm{~cm}$ and the white arrow indicates uterus. Uterus size was not measured because of huge size uterus

In October 2012, the patient opted for laparotomy myomectomy for recurrent uterine fibroids. A total of 80 intramural uterine fibroids were removed during surgery with total estimated blood loss of 6 litres. The patient required ICU admission with a total of 6 pints pack cells, 4 units of fresh frozen plasma, and 6 units cryoprecipitate were transfused. 
The patient recovered well. Histopathology confirmed leiomyomas (Figure 2). After 3 months of post myomectomy, uterus size was $9 \times 4 \mathrm{~cm}$ (Figure 3 ).

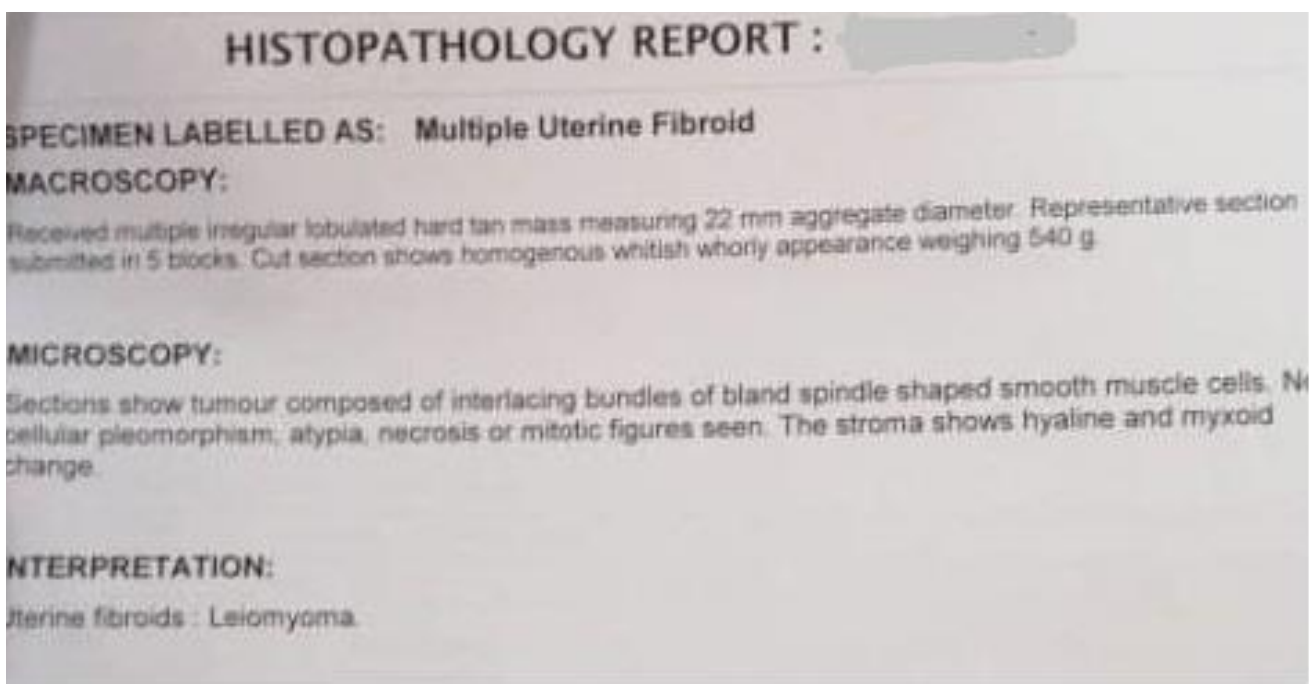

Figure 2 Histopathology report

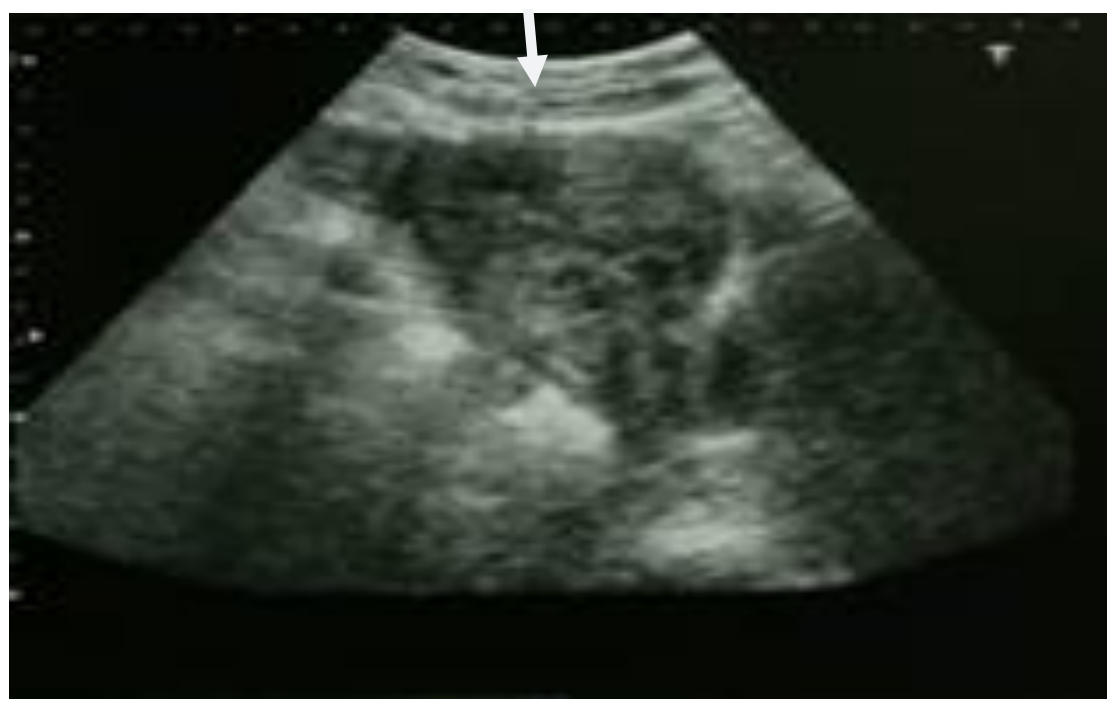

Figure 3 Three months of post myomectomy. Arrow indicates uterus approximately $9 \times 4 \mathrm{~cm}$

In 2013, it was noted asymptomatic small uterine fibroids which might be missed during previous laparotomy myomectomy, however, patient was not keen for any active management as her lifestyle was much improved after myomectomy where she experienced significant improvement of dysmenorrhea, no prolonged or heavy menstrual bleeding, no more hospital admissions for blood transfused, active and can do her duty until last review 2016. The patient has opted for conservative management as she would decide for her fertility workup after she was counselled and opted for conservative treatment by fertility clinic team at a tertiary centre in July 2011. The patient did not turn up during follow-up at the fertility clinic after 2011. After her last myomectomy surgery was done in October 2012, her quality of life has been improved for the following years. She did not have heavy menstrual bleeding while she was able to resume her work without any complaint of anaemia or dysmenorrhea and she did not need to be transfused as before the last myomectomy surgery. 


\section{DISCUSSION}

Uterine fibroids (also known as leiomyomas or myomas) are the commonest benign uterine tumours, with an estimated incidence of 20 - $40 \%$ in women during their reproductive years ${ }^{3}$. Victor Bonney was the pioneer surgeon introduced myomectomy in the history of gynaecological surgery in the 1920s. Most myomas start as asymptomatic and diagnosed during a routine pelvic examination. Myoma starts small and gradually increasing in size until it becomes symptomatic causing heavy menstrual bleeding and dysmenorrhea and might be associated with infertility. Current recommendations include consideration of myomectomy in infertile women after extensive evaluation eliminating other causes of infertility ${ }^{4}$. The uterine fibroid risk factor with the strongest evidence is black race $^{5}$. Active management as myomectomy is the treatment of choice among women with heavy menstrual bleeding as compared to conservative management especially in the terms of preserving fertility during childbearing age. Before the advent of laparoscopic technique in myomectomy procedure most surgeries were done through a laparotomy approach, which is considered an invasive surgery but still one of the conventional techniques for myomectomy procure until today. It is a recommended operation for those cases with contraindication for minimally invasive surgery. When expertise for laparoscopic myomectomy is available, open myomectomy is usually reserved for women who have multiple or extremely large fibroids ${ }^{6}$. Patient's quality of life has been tremendously improved post myomectomy operation in October 2012. She did not complain of heavy menstrual bleeding or symptoms of anaemia. She was able to commence her daily life without needing blood transfusions or complaining of dysmenorrhea as before her last myomectomy operation.

\section{CONCLUSION}

Patient with clinical pictures of severe dysmenorrhea, heavy menstrual bleeding and poor quality of life due to multiple uterine fibroids can be improved tremendously after myomectomy operation.

\section{ACKNOWLEDGEMENTS}

The authors would like to thank the patient for her cooperation in relation to the writing of this case report. The author would also like to thank the Director of Hospital Labuan.

\section{CONFLICT OF INTEREST}

The authors declare that they have no competing interests in publishing this case.

\section{CONSENTS}

Written informed consent was obtained from the patient to publish the case. A copy of the written consent is available for review by the Chief Editor.

\section{REFERENCES}

1. Mariagiulia B, Lucia L, Federica P et al. (2017). Dysmenorrhea and related disorders. Version 1. F1000Res 6: 1645. PMID:28944048

2. Karim AC. (2018). Dysmenorrhea. Medscape, Drugs \& Diseases, O\&G 2018. Available from: https://emedicine.medscape.com/ article/253812-overview\#a4

3. Aamir TK, Manjeet S, Janesh KG. (2014). Uterine fibroids: current perspectives. Int J Women's Health 6: 95 - 114. PMID: 24511243. Available from: https://www.ncbi.nlm.nih. gov/pmc/articles/PMC3914832/ 
4. Sarah HM. (2018). Gynecologic myomectomy treatment and management. Medscape, Drugs \& Diseases, Clinical Procedures. Available from: https://emedicine. medscape.com/article/267677-treatment

5. Stewart EA, Cookson CL, Gandolfo RA, Schulze-Rath R. (2017). Epidemiology of uterine fibroids: A systematic review. BJOG: An International Journal of Obstetrics and Gynaecology 124 (10): 1501 - 1512. DOI: https://doi.org/10.1111/1471-0528.14640
6. Saridogan E. (2016). Surgical treatment of fibroids in heavy menstrual bleeding. Womens Health (Lond) 12 (1): 53 - 62. Epud 2015 Dec 23. PMID: 26693796 DOI: 10.2217/ whe.15.89. 
